\title{
THE NASA LANGLEY RESEARCH CENTER'S UNMANNED AERIAL SYSTEM SURROGATE RESEARCH AIRCRAFT
}

\author{
Charles T. Howell III, NASA Langley Research Center, Hampton, VA; Artie Jessup, Science \& \\ Technology Corp., Hampton, VA; Frank Jones, NASA Langley Research Center, Hampton, VA; Claude Joyce, \\ ATK Space Systems In., Hampton, VA; Paul Sugden, Unisys Corp. Hampton, VA; Harry Verstynen, Unisys \\ Corp., Hampton, VA; and John Mielnik, Lockheed Martin Corp., Hampton, VA
}

\begin{abstract}
Research is needed to determine what procedures, aircraft sensors and other systems will be required to allow Unmanned Aerial Systems (UAS) to safely operate with manned aircraft in the National Airspace System (NAS). The NASA Langley Research Center has transformed a Cirrus Design SR22 general aviation (GA) aircraft into a UAS Surrogate research aircraft to serve as a platform for UAS systems research, development, flight testing and evaluation. The aircraft is manned with a Safety Pilot and systems operator that allows for flight operations almost anywhere in the NAS without the need for a Federal Aviation Administration (FAA) Certificate of Authorization (COA). The UAS Surrogate can be controlled from a modular, transportable ground station like a true UAS. The UAS Surrogate is able to file and fly in the NAS with normal traffic and is a better platform for real world UAS research and development than existing vehicles flying in restricted ranges or other sterilized airspace.
\end{abstract}

The Cirrus Design SR22 aircraft is a small, singleengine, four-place, composite-construction aircraft that NASA Langley acquired to support NASA flight-research programs like the Small Aircraft Transportation System (SATS) Project. Systems were installed to support flight test research and data gathering. These systems include: separate research power; multi-function flat-panel displays; research computers; research air data and inertial state sensors; video recording; data acquisition; data-link; S-band video and data telemetry; Common Airborne Instrumentation System (CAIS); Automatic Dependent Surveillance-Broadcast (ADS-B); instrumented surfaces and controls; and a systems operator work station. The transformation of the SR22 to a UAS Surrogate was accomplished in phases. The first phase was to modify the existing autopilot to accept external commands from a research computer that was connected by redundant data-link radios to a ground control station. An electro-mechanical auto-throttle was added in the next phase to provide ground station control of airspeed. Additional phases are in progress to add waypoint navigation and long range satellite voice and data communications. Potential areas for UAS Surrogate research include the development, flight test and evaluation of sensors to aid in the process of air traffic "detect-sense-and-avoid". These sensors could be evaluated in real-time and compared with onboard human evaluation pilots. This paper describes the systems and design considerations that were incorporated in the development of the UAS Surrogate along with details of development problems encountered and the corresponding solutions.

\section{Introduction}

The NASA Langley Research Center has a long history of aeronautics research. In 2001, three GA aircraft were procured including a Cirrus Design SR22 for use as research aircraft. These aircraft were used in a variety of research programs including the NASA SATS Program. As a result of the research programs, many different research support systems were installed in these aircraft. These systems included computers, display, telemetry, power, data acquisition and other research systems. When the research programs ended, the aircraft became available for new research. The idea of a UAS surrogate was developed in house. The UAS Surrogate would make use of many of the research support systems already installed in the aircraft. A proposal was submitted to NASA Langley Senior Management to use the Cirrus SR22 aircraft as the basis for the UAS Surrogate and it was accepted. The SR22 was chosen because it had the most desirable 
installed research systems. These systems included a separate alternator and power system, three research computers, a data acquisition system, S-Band telemetry, multi-function display (MFD), video recording, and fully instrumented engine and control surfaces. The NASA Langley Cirrus SR22X aircraft, N501NA, is shown in Figure1.

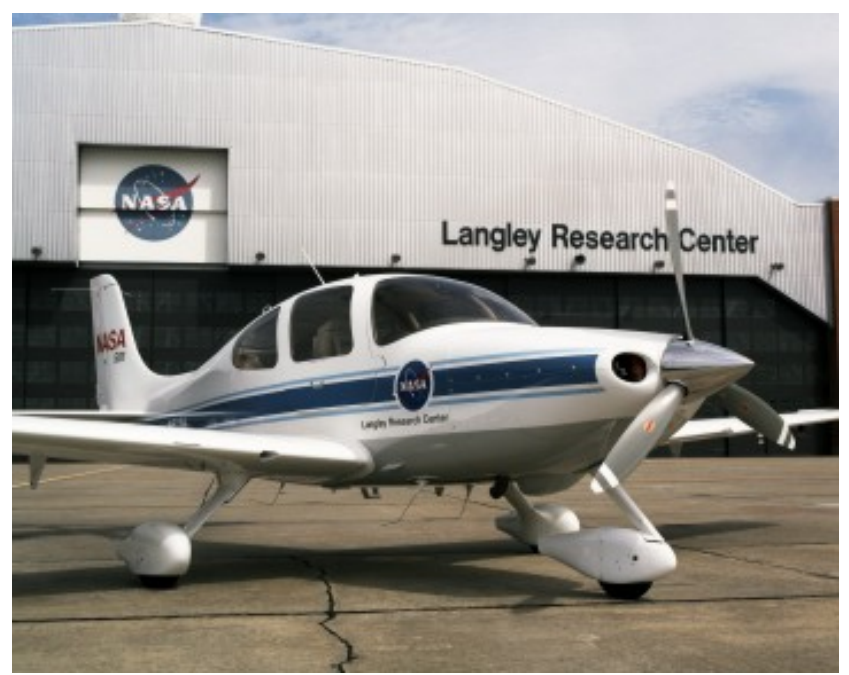

Figure 1. Cirrus Design SR22 Aircraft

\section{The Cirrus Design SR22 Aircraft}

The basis for the UAS Surrogate is the Cirrus Design SR22 general aviation aircraft. The Cirrus SR22 is a composite construction, single-engine, four-place, GA aircraft manufactured by Cirrus Design of Duluth, Minnesota. The SR22 is one of several new-generation GA aircraft making use of the latest in materials, aerodynamics, avionics, and manufacturing technology. The SR22 aircraft received Federal FAA certification in 2000, with hundreds of aircraft having been delivered since that time. One of the innovative design features of the SR22 aircraft is the Cirrus Airframe Parachute System (CAPS). The CAPS is an emergency parachute system that can be deployed by the pilot or a passenger to safely slow and lower the entire aircraft to ground if controlled flight is no longer possible.

The aircraft design makes use of single-handed side control instead of traditional two-handed control yokes. The aircraft has an empty weight of $2512 \mathrm{lbs}$, a gross weight of $3400 \mathrm{lbs}$, a load capacity of $988 \mathrm{lbs}$, and a fuel capacity of 81 gal (486 lbs). The engine is a 310-hp Teledyne Continental model IO-550-N with 6 cylinders, fuel injection, and is normally aspirated. The SR22 aircraft has a wingspan of 33-ft, length of 26- $\mathrm{ft}$, and a height of 8-ft. Performance specifications include a cruise speed of 180 knots, climb rate of $1400 \mathrm{ft} / \mathrm{min}$, takeoff roll of $1100-\mathrm{ft}$, and a ceiling of $17,500-\mathrm{ft}$.

As delivered to NASA, the aircraft avionics suite includes: dual Garmin GNS 430 Global Positioning System (GPS)/communication/navigation units; STEC 55X autopilot; Garmin GTX 327 Mode-C Transponder; Garmin GMA 340 Audio Panel; Sandel Electronic Horizontal Situation Indicator (EHSI); digital altitude encoder; L-3 (Goodrich) WX-500 Stormscope; and, an ARNAV 10.4-in. diagonal flatpanel MFD. The original ARNAV display was replaced with the Avidyne FlightMax EX5000 MFD. The GNS 430 units are certified for Instrument Flight Rule (IFR) approaches, and include the following components: Very-High Frequency (VHF) communications transceivers, and receivers for GPS, VHF Omnirange (VOR), Instrument Landing System (ILS), Glide Slope, and Marker Beacon. The production avionics systems also include an emergency locator transmitter (ELT). Traditional "steam" gauges include: indicated airspeed indicator; electric attitude indicator; altimeter; turn coordinator; vertical speed indicator; GPS/ILS indicator; magnetic compass; fuel quantity gauges, manifold pressure/fuel flow gauge; oil temperature/oil pressure gauge; Exhaust Gas Temperature-Cylinder Head Temperature (EGT/CHT) gauge; and, voltage/amperage gauge.

\section{Aircraft Modifications}

\section{Standard Autopilot}

The Cobham S-TEC 55X Autopilot is a standard two axis (pitch and roll) autopilot used in several GA aircraft. The avionics stack-mounted case contains the autopilot mode selector, programmer, annunciator, roll and pitch computers, and servo amplifiers. The S-TEC 55X has several modes of operation including Heading (HDG), Navigation (NAV), Approach (APR), Reverse (REV), Altitude Hold (ALT), Vertical Speed (VS) and GPS Steering (GPSS). It was initially decided that the desired level of aircraft remote control could be achieved by 
controlling the aircraft's heading, altitude, and vertical speed via the autopilot. This approach takes advantage of the built in safety features of the autopilot including the ability to fly the aircraft but allow the pilot to override or disengage the system. Airspeed is handled by directly driving the aircraft throttle handle through a connecting rod with a separate dedicated servo.

\section{Autopilot Modifications}

By examining how the autopilot was designed and manufactured, it was determined that it could be easily modified to allow for external mode control as well as vertical speed control. A duplicate S-TEC $55 \mathrm{X}$ autopilot control panel was purchased for modification in order to preserve the certified unmodified unit. The modified autopilot is shown in Figure 2.



Figure 2. Modified Autopilot

It was determined that the six mode switches could be controlled by external ground-open discrete signals from a computer in parallel with the existing front panel push button controls. Wires were added to the internal circuit board for each switch and brought outside the autopilot via unused connections on the 50-pin connector on the back of the unit. The 3discrete output rotary switch controlling the vertical speed on the 55X front panel was different in that it could not be wired in parallel. The autopilot internal rotary switch connections were severed and external wires were added and brought out of the autopilot from unused rear pins as substitutes for the rotary switch. This modification permanently disabled the vertical speed control from the rotary switch on the autopilot front panel, but allowed control from the research computer discrete outputs.

When the aircraft software operator has been given clearance from the Safety Pilot, he/she enables the software control of the aircraft. At this point, the software manipulates discrete outputs of the Condor CEI-520 card in the research computer to simulate button presses required to transition the S-TEC-55X into GPSS mode, and then into ALT mode. The software also begins sending zero roll commands and the current ground speed required for GPSS mode via the CEI-520 Aeronautical Research Incorporated (ARINC) 429 output serial bus.

When a vertical speed command is issued, the Condor CEI-520 discrete outputs are used to simulate a VS mode button press, followed by an appropriate number of simulated vertical speed knob clicks to achieve the commanded vertical speed. An actual pilot would look at the front panel of the S-TEC-55X after transition to VS mode, and make the number of clicks required to close the difference in the displayed value, and the desired value. Currently, the research computer has no feedback from the S-TEC$55 \mathrm{X}$ to verify the selected vertical speed. This limitation necessitated incorporation of various heuristics for predicting the actual value, and silent generation of subsequent simulated Vertical Speed knob clicks if the predicted value does not match the command. These heuristics have been shown to perform well from smooth air to moderate turbulence.

The S-TEC 55X autopilot normally receives navigation steering commands from the Garmin GNS 430 GPS navigation system. An external switch was installed on the cockpit center console to allow the Safety Pilot to switch the source of the ARINC 429 steering signal inputs to the autopilot. The "Normal" switch position connects the \#1 Garmin GNS 430 steering signals to the autopilot; and, the "Research" UAS position connects the research computer to the autopilot steering signal input. The Condor CEI-520 ARINC 429 interface card in the research computer and the associated software provide the steering signals to the autopilot. 
When a heading command is issued, the ARINC 429 roll command that is being sent to the autopilot becomes proportional to the difference in commanded and actual heading, but is limited to approximate a standard rate turn. The periodic transmission of the current ground speed continues, as this parameter is required by the autopilot to provide an appropriate level of roll authority.

\section{Autothrottle System}

The autothrottle was designed to be simple, safe, and removable. The autothrottle system components include a modified roll servo, a drive rod connected to the throttle handle, an in-house fabricated servo amplifier, and a commercial digital-to-analog converter. A lever arm was fabricated for the servo to hold the drive rod. The drive rod is connected to the servo lever at one end and the throttle lever at the other end. The rod is held in place by easy to remove cotter pins located at both ends. The autothrottle system can easily be disabled by removing one or both cotter pins and removing the throttle drive rod. The autothrottle servo is mounted to a removable structure located on the rear of the center console and anchored to the rear seat mounting pins. The servo amplifier and digital-to-analog converter are mounted to the top of the general purpose computer that is located where the left rear seat is normally installed.

The servo has an adjustable clutch to allow setting a specific amount of friction to operate the throttle but also allow the Safety Pilot to manually override the servo. The clutch friction was set to provide 41.3 in. lbs of torque. This setting provided the force needed to drive the throttle full forward, full aft, and the force to overcome the propeller pitch gate detent. The servo moves at an angular rate of 4.9 deg per sec with a stop-to-stop travel of $\pm 20 \mathrm{deg}$. The servo has an electrical clutch engage system with a switch mounted on the center console that allows the Safety Pilot to control this function. In order to operate the autothrottle, the Safety Pilot must engage the switch and the software operator must enable. Disabling either will disable the system. An autothrottle system block diagram is shown in Figure 3.

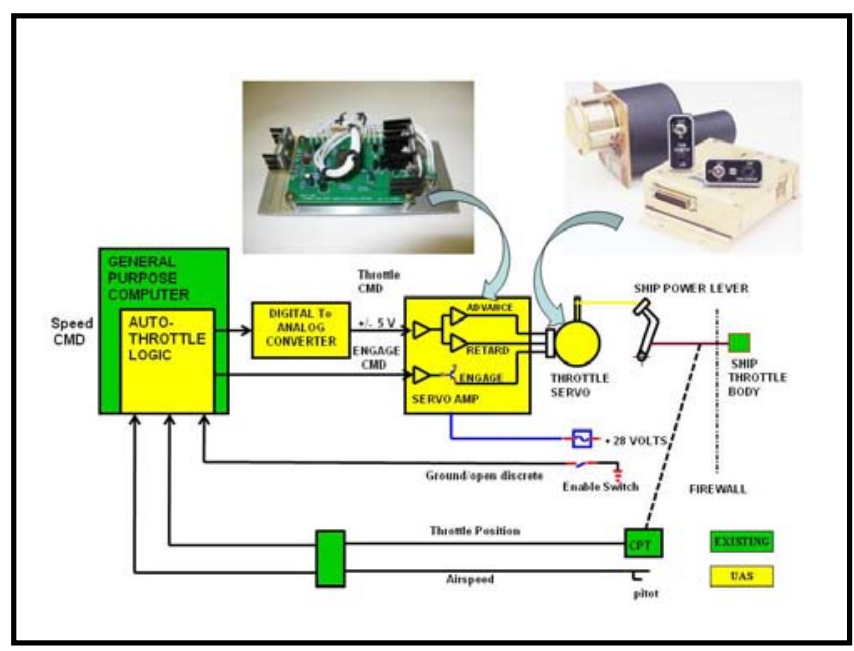

Figure 3. Autothrottle System Diagram

\section{Roll Servo Modification}

Flight tests revealed that the research autopilot had reduced authority above 148 knots. The aerodynamic forces on the ailerons were greater than those generated by the autopilot. The problem was caused by the limited authority of the autopilot Roll Trim Cartridge. The Roll Trim Cartridge implementation on the SR22 is an alternative to a dedicated roll servo. Due to several limitations of the roll trim cartridge and other autopilot problems, STEC developed a Supplemental Type Certificate (STC) modification for a dedicated roll servo upgrade for the Cirrus SR22. The servo upgrade was designed specifically for the S-TEC 55X autopilot and the Cirrus SR22 aircraft. This upgrade was designed to improve autopilot roll authority, navigation tracking, ILS localizer capture, operation in turbulence, and operation at higher speeds. Flight tests performed with the un-modified autopilot after the roll servo upgrade modification confirmed that the autopilot had improved roll channel performance. Improvements were demonstrated with navigation tracking, ILS captures, and control at speeds up to the SR22 never exceed speed. The aircraft is awaiting further UAS Surrogate flight tests to assess the impact of the servo upgrade on the research autopilot.

\section{Components/Architecture}

There are many existing aircraft system elements that were installed during the SATS program prior to the UAS Surrogate Project. These include the CAIS, video cameras, video tape recorders, S-band 
telemetry system, the air data and heading reference air data and heading reference system (ADAHRS), fully instrumented controls, and a universal access transceiver (UAT). The UAT can provide ADS-B and other information. The aircraft hardware architecture is shown in Figure 4.

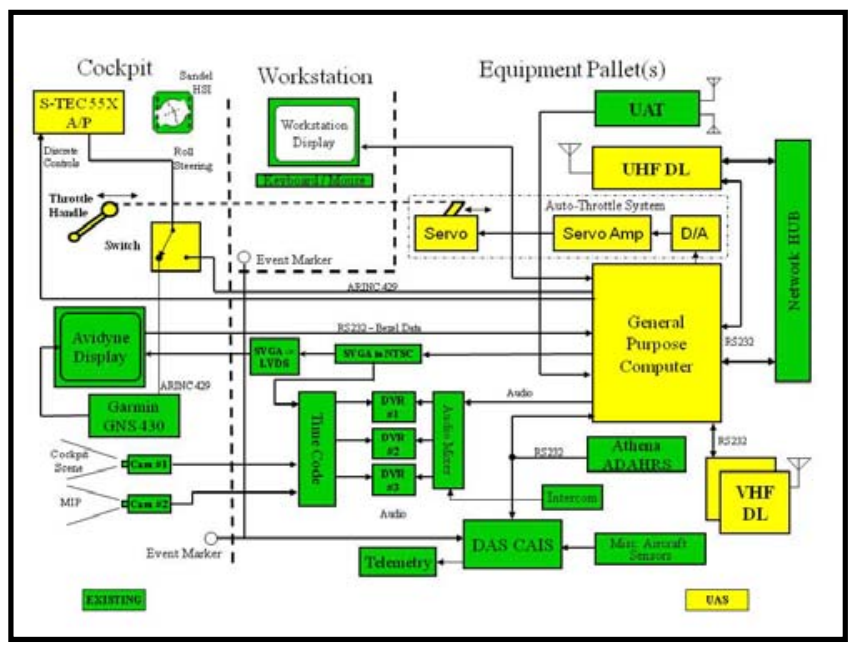

Figure 4. UAS System Architecture

The aircraft computer system consists of an Intel dual-core processor-based PC running the Microsoft Windows XP operating system. The computer is connected to the VHF and Ultra High Frequency (UHF) radios via the RS-232 serial interface at 9600 baud. The computer also gets air data, inertial, and GPS data from the Athena GS-111m ADAHRS system over an RS-232 interface at 57,600 baud. The ADAHRS unit provides all data updates at $50 \mathrm{~Hz}$. The computer also receives bezel button and rotary knob data from the Avidyne FlightMax EX5000 MFD over an RS-232 interface. The Avidyne display system was modified by the manufacturer to provide "Normal" and "Research" display modes. In the Normal Mode, the Avidyne system provides the standard FAA-certified moving map and other data. An instrument panel switch allows changing to the Research Mode where the custom display images are provided from the research computer system and software. The Condor CEI-520 card provides the ARINC 429 and discrete interface to the autopilot as previously described. The UAT is interfaced to the computer by an RS-422-to-Universal Serial Bus (USB) converter.

\section{Radio Data-Link System}

The original data-links used on the Cirrus were Pacific Crest RFM96, 2-W, 4800-baud, VHF (150$175 \mathrm{MHz}$ ) radio modems originally used for differential GPS applications. Testing proved that the throughput, power and range of these radios proved to be inadequate for the UAS Surrogate application. It was determined that for the UAS application, a minimum of 9600 baud was required to send a 50100 byte message several times per second. A maximum operating range from the ground station of 30-40 n. mi, was desired.

\section{Spread Spectrum UHF Radios}

The first of two replacements for the original radio modems are the FreeWave FGRPlus, 1-W, license free, $900-\mathrm{MHz}$, spread spectrum radios. These spread spectrum radios have more than the required throughput with additional capabilities including: dual RS-232 channels; repeater capabilities; maximum throughput of 154,000 bits/sec; Ethernet; and User Datagram Protocol (UDP) communications. However, the reliable range proved to be limited to about $10 \mathrm{n}$. mi. in flight tests with the $1-W$ transmitter power. This reduced range is believed to be due in part to the composite aircraft construction (lack of metal ground material found in most metal aircraft), and an inefficient antenna. The result is poor radio range performance. Efforts to improve the range and reliability are ongoing. The latest effort involves the purchase of external amplifiers to boost the transmitter power to 5-W output with a better aircraft antenna. Further flight testing is required to test these modifications.

\section{VHF Radios}

The second replacement for the original radios is the addition of Teledesign TS4000 VHF radios. These programmable radios operate in the 150-175$\mathrm{MHz}$ band, output a maximum of $5-\mathrm{W}$, and have a signaling rate of up to $19,200 \mathrm{bits} / \mathrm{sec}$. Two sets of these radios, two in the Ground Station and two in the aircraft, are used to provide redundancy. Two frequencies spaced $5 \mathrm{MHz}$ apart are currently authorized and provide redundancy and frequency separation. The two Teledesign VHF radios are used in addition to the Freewave UHF radios for both redundancy and diversity. Currently, only two radios 
are used at a time, two TS4000 radios or one FGRPlus and one TS4000 radio. The software can be configured to use all three radios simultaneously.

\section{Data-Link Problems}

Even though two frequencies were authorized for use in the $162-174-\mathrm{MHz}$ range, this is a very congested area of the spectrum with more frequency assignments and equipment usage than any other 12$\mathrm{MHz}$ band allocated to the Federal Government [1]. Users of this band include Government Land Mobile Operations such as Police, Fire, Local Government, Marine Band, Business Band, NOAA Weather Radio Broadcast and others. As a result, interference was encountered during flight tests. The interference was found to be worse in certain locations and the mitigation strategy was to avoid those areas when possible. This situation points to a problem for UAS operations in general and a topic for further research how to provide dedicated reliable, redundant, communications to and from UAS's and their respective ground stations.

\section{Antenna Efficiency}

Although the TS4000 radios generally have good performance and capabilities, many problems were encountered with the process of sending messages between the aircraft and ground station. Most of the problems were with the aircraft. The problems start with the composite construction of the aircraft. Most commercially-available VHF or higher frequency aircraft antennas are designed to use the aircraft's metal skin to approximate an infinite ground plane to improve the efficiency and performance [2]. The SR22 and most similar commercially-available composite aircraft are delivered with antennas designed for metal aircraft. Consequently, many of these aircraft suffer from reduced performance of their radio frequency systems. For this reason the standard VHF voice communications radios on the SR22 have demonstrated a maximum air-to-ground range of about $30-40 \mathrm{n}$. mi. at an altitude of $3500 \mathrm{ft}$. The theoretical line of sight range is close to $100 \mathrm{n}$. mi.

\section{Radio Interference}

Radio interference was also discovered from several sources. Ground and airborne tests with a spectrum analyzer revealed that the aircraft VHF voice communications radios were interfering with the VHF TS4000 data-link radios but not the reverse. This situation was found to be true even though the aircraft radios operate only in the 118.00 to 136.00 $\mathrm{MHz}$ frequency band and the data-link radios operate in the 150.00 to $175.00-\mathrm{MHz}$ range. As a result of this discovery, customized band pass filters were purchased to eliminate this interference. The K\&L Microwave 3B110-167/T10-B/BP filters were designed to pass frequencies within $5 \mathrm{MHz}$ of the desired center frequency and attenuate or reject all others. Airborne tests also revealed relatively highpowered ground transmitters operating very close to the authorized data-link frequencies that were potential sources of interference. The band pass filters and physically avoiding areas close to subject transmitters when possible mitigated most of the radio interference.

\section{Electromagnetic Interference (EMI)}

EMI has been a continual problem with the research systems on the Cirrus SR22. EMI is another issue with composite aircraft in general because of the lack of metal ground material. Also, the metal skin of traditional aircraft serves as a Faraday shield that protects internal systems from external interference and is absent from most composite constructed aircraft [3]. The metal skin also serves as a ground plane for various antenna types and an electrical grounding point for electrical systems and a means to mitigate internal EMI.

One manifestation of EMI problems is noise heard in the aircraft VHF communications radios on one or more of the frequencies between 118.00 and 136.00 MHz. This noise comes from several research system sources including the research computer, video signals, data bus signals, and other computer interface signals. For this reason, all VHF frequencies are checked for EMI after any significant changes to the research electronics systems. The mitigation for most of the EMI problems encountered on the SR22 aircraft is proper grounding and shielding. Covering any unshielded cables with overbraid is another EMI mitigation strategy that was successfully used. Ovebraid is a flexible metal conduit manufactured with conductive metal wire over braiding. When used as an outer cover for existing cables and electrically 
grounded at one or both ends, overbraid is a proven EMI mitigation technique. To improve grounding, all aircraft electrical grounds are also tied directly or indirectly to the aircraft motor mount, which is the largest metal structure in the composite aircraft. Another EMI mitigation technique was the use of EESeals. EESeals are commercially available thin flat capacitance filters designed to be placed in-line with existing standard connectors. These filters were effectively used on the computer interface signal connectors including RS-232 and video to reduce EMI.

\section{Antennas}

With the poor performance of the commercial VHF and UHF antennas, it was decided to build antennas that were less dependent on the use of metal ground planes. Research determined that one of the oldest and simplest antennas is the dipole antenna which generally does not require a ground plane to be efficient [4]. Several half-wave dipole antennas were constructed for testing using 50-ohm coaxial cable, ferrite beads, and 2-in. wide copper foil tape. Standard wavelength calculations were made using standard formulas [5]. For the operating frequency of $167 \mathrm{MHz}$, the wavelength is $1.796 \mathrm{~m}$ or $70.71 \mathrm{in}$. and less than 2-in. longer for the $162.00 \mathrm{MHz}$ frequency. The $1 / 4$ wavelength dimension is 17.68 inches and the two $1 / 4$ wavelength sections make the $1 / 2$ wave dipole antenna. For $915 \mathrm{MHz}$, the center frequency of the spread spectrum radio band, the dimensions are much shorter at $6.4525 \mathrm{in}$. for the $1 / 4$ wavelength dimension.

\section{Dipole Antenna Fabrication}

The VHF half-wave dipole antennas were constructed by stripping the shield and center conductor of the 50-ohm coaxial cable back a sufficient distance to solder each to an 18-in. length of copper foil tape. The 18-in. length was chosen to be longer, or lower in center frequency, than desired. The longer length allows for tuning by cutting the length to achieve the desired higher frequency. Ferrite beads were inserted at the meeting point between the coaxial cable and the copper tape. The ferrite beads act as a transformer to match the 50- ohm coaxial cable impedance to the approximately 377-ohm free space impedance [6]. Both copper tape sections were conformally mounted to the outside of the aircraft. The coaxial cables were run to openings in the fuselage and connected to the radios. Speed tape was used to cover and hold the vulnerable parts. The VHF Antenna \#1 was folded over the vertical tail surface and VHF Antenna \#2 was mounted vertically on the left fuselage side. The antennas were then tested and tuned to the desired center frequencies of 162.00 and $167.00 \mathrm{MHz}$. These same techniques were used to build $900-\mathrm{MHz}$ antennas; however the results are inconclusive due to the lack of time and test equipment for this frequency band.

\section{Antenna Tuning}

Tuning the half-wave dipole antennas involved measuring the antenna voltage standing wave ratio (VSWR), the S11 two-port network scattering parameters, and the reflected power using several pieces of test equipment. The S11 is the input port voltage reflection coefficient and is measured with a network analyzer at the center frequency. The $1 / 4$ wave antenna elements were iteratively shortened by cutting small 1/8-in. sections from each end, then testing until the reflections were minimized. It was determined through trial and error that tapering the ends of the foil tape also had an effect on the S11 measurement as well as the flatness of the frequency range being tested. Another method used in the antenna fabrication and on-aircraft tuning was the utilization of a spectrum analyzer and directional coupler. This method essentially measured the reflected power (VSWR) of the antenna. The internal tracking source was utilized in the spectrum analyzer for the input into the antenna through the directional coupler. The reverse power port of the coupler was then connected to the input of the spectrum analyzer. The antenna was tuned and positioned to minimize the reflected power and for the flatness of the operating frequency range. Factors affecting the tuning process were the spacing between the elements, the angular orientation between the elements, and structures within the aircraft. It was also determined that coaxial cable routing had an effect. The two conformal VHF aircraft antennas are shown in Figure 5. 


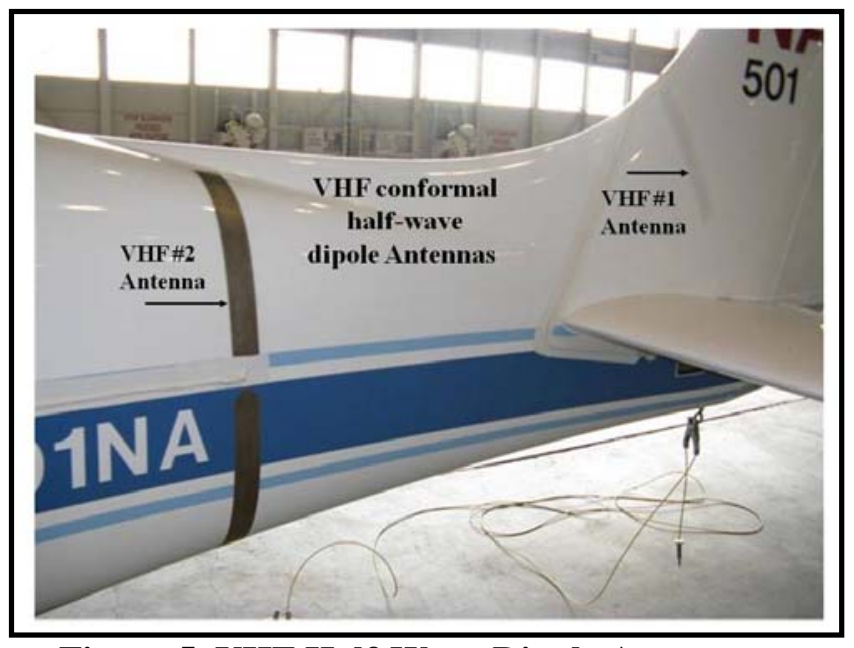

Figure 5. VHF Half-Wave Dipole Antennas

After tuning, the $1 / 4$ wavelength sections of the tuned antennas measured about 14.5 in., or about 3 in. less than theoretically calculated values. Ideally, a VSWR of 1.0 is desired and any value below 1.5 is considered adequate. This process yielded VSWR numbers slightly below 1.1 at the respective center frequencies. For comparison, typical VSWR numbers between 1.5 and 2.5 were obtained for the commercial blade and whip antennas installed on the aircraft and some that were later purchased. These antennas were designed for metal aircraft and they have demonstrated reduced performance on composite aircraft.

\section{Software System}

The surrogate UAS is operated from a PC-based Ground Station which presents the operator with a moving map having a sectional chart as the background, a primary flight display (PFD), and a graphical user interface (GUI) for entering altitude, vertical speed, heading, and speed commands. The aircraft software includes identical moving map and PFD, and a similar GUI. The moving map is presented to the Safety Pilot on the Avidyne MFD when the display is switched to the Research Mode, allowing the Safety Pilot to monitor commands as they are received from the Ground Station. In addition, the research software operator is presented with the PFD, and a GUI that is an extended version of Ground Station GUI. All UAS software was developed using the Langley Standard Real-time Simulation in $\mathrm{C}++(\mathrm{LaSRS}++)$ software development and test facilities.

\section{Graphical User Interface (GUI)}

The Ground Station UAS Control GUI has four panels for entering Vertical Speed, Altitude, Heading, and Airspeed Commands. An "OFF / ON" box is also included to control individual commands. Command numbers may be changed with a mouse and left clicking the respective "+” or "“" buttons. The operator may also left click inside one of the respective command boxes and enter a number from the keyboard. A control option for frequency of updates from the aircraft is provided. There are also indicators for the number command transmissions from the Ground Station, the number of received messages from the aircraft, the number message CRC errors, the number of message bytes skipped, and the aircraft GPS Time. Other GUI panels are optionally available to the Ground Station operator to display additional data and status information. The Ground Station Control GUI is shown in Figure 6.

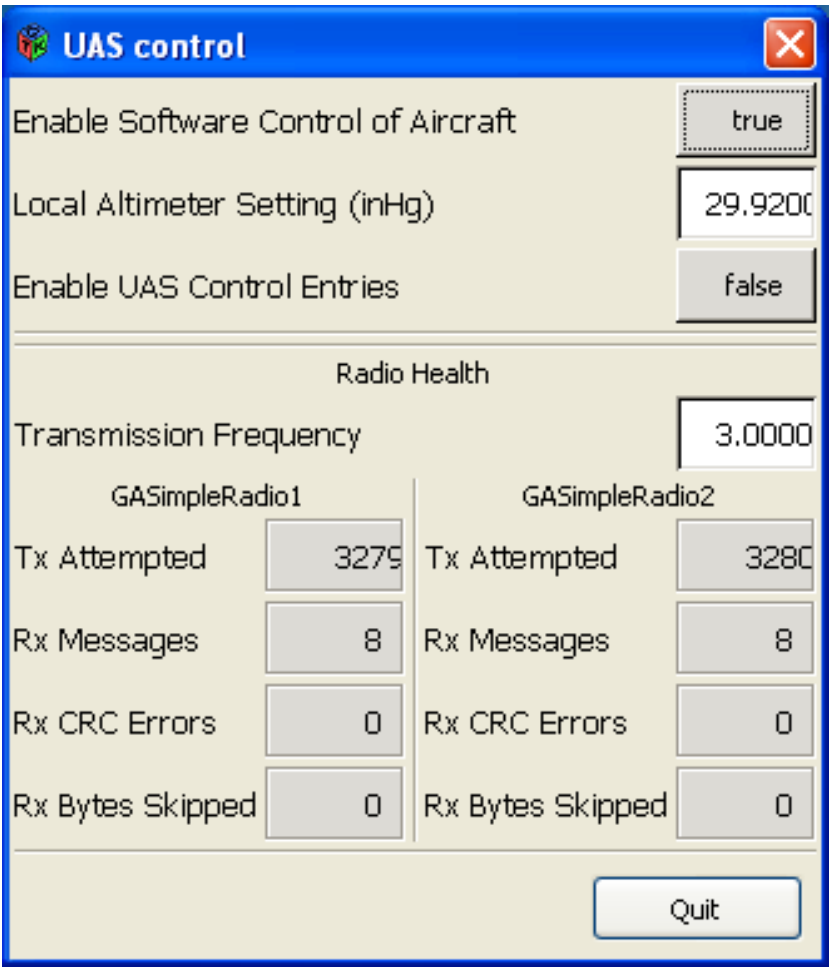

\section{Figure 6. Ground Station Control GUI}

A similar UAS Control GUI is also displayed to the Systems Operator in the aircraft. This GUI has a few additional controls. A "Enable Software Control of Aircraft" button is used to enable or disable any software commands. When "Enable UAS Control Entries" is selected, command entries may be made 
from the Operator's Work Station on the aircraft, and are disabled at the Ground Station. A "Local Altimeter Settings" box allows the Systems Operator to set the current altimeter setting. The remainder of the GUI is the same as for the Ground Station. Several optional GUI panels are also available to the Systems Operator for the display and control of several software parameters. These additional GUI panels allow the Systems Operator to change gains, time constants and other control law and filter related parameters. The aircraft UAS Control GUI is shown in Figure 7.

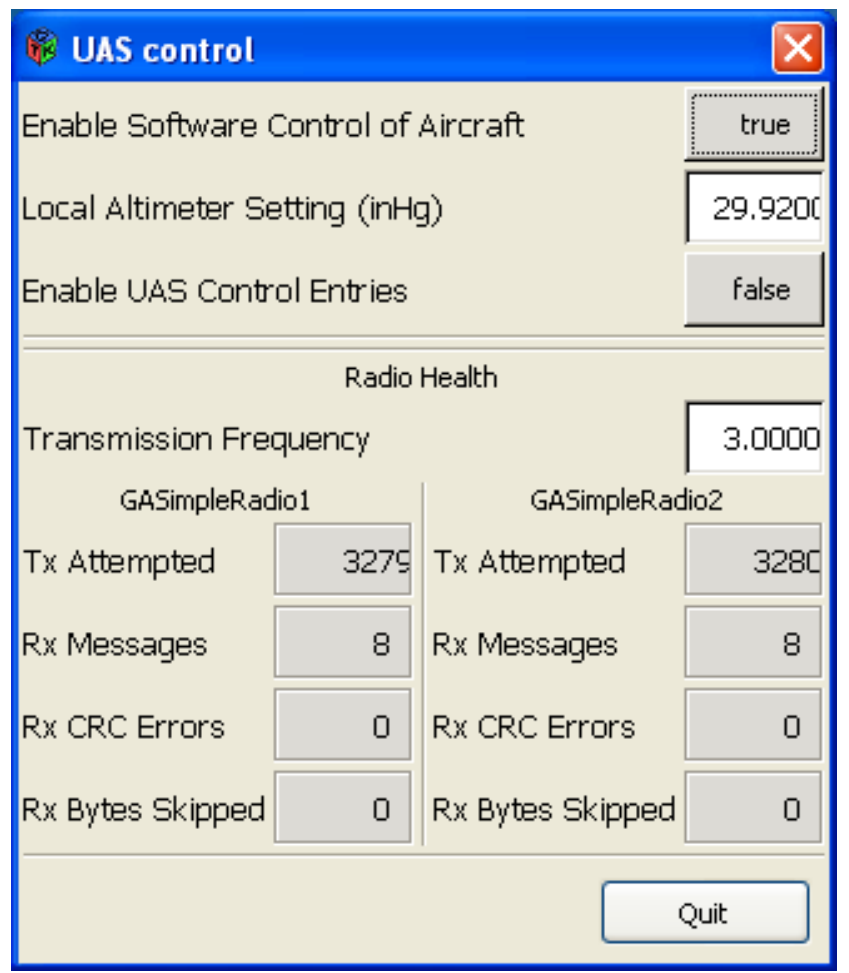

Figure 7. Aircraft Control GUI

\section{Moving Map Display}

The Moving Map Displays are identical in the aircraft and Ground Station with independent control of brightness, declutter, North-Up/Track-Up and map range. All four of the active Ground Station Commands are shown in lower left box on the display. This allows both the Safety Pilot and the Ground Station Operator to verify and confirm the commands. A box in the upper left shows the current ground speed, indicated airspeed, true heading, wind speed, and wind direction from the ADAHRS. An upper middle box shows the current magnetic heading. Current ADAHRS GPS data is displayed in an upper right box that includes GPS satellites tracked, position dilution of precision (PDOP), time, and GPS Mode. Map range is displayed in a middle bottom box and relative display brightness is on the bottom right. The map background is a georeferenced FAA Sectional Aeronautical Chart. The current aircraft position is shown by an aircraft symbol in the middle of the Moving Map Display. A 30-60-90-sec trend vector is also attached to the aircraft symbol and shows where the aircraft is projected to be at those times. The current aircraft latitude, longitude, and altitude parameters are displayed near the aircraft symbol. A compass rose is displayed showing the current aircraft heading with green triangle. The map is user selectable for North-Up or Track-Up display modes. A line from the aircraft symbol to the compass rose shows the magnetic track and a magenta chevron shows the commanded heading. Figure 8 shows the moving map display.

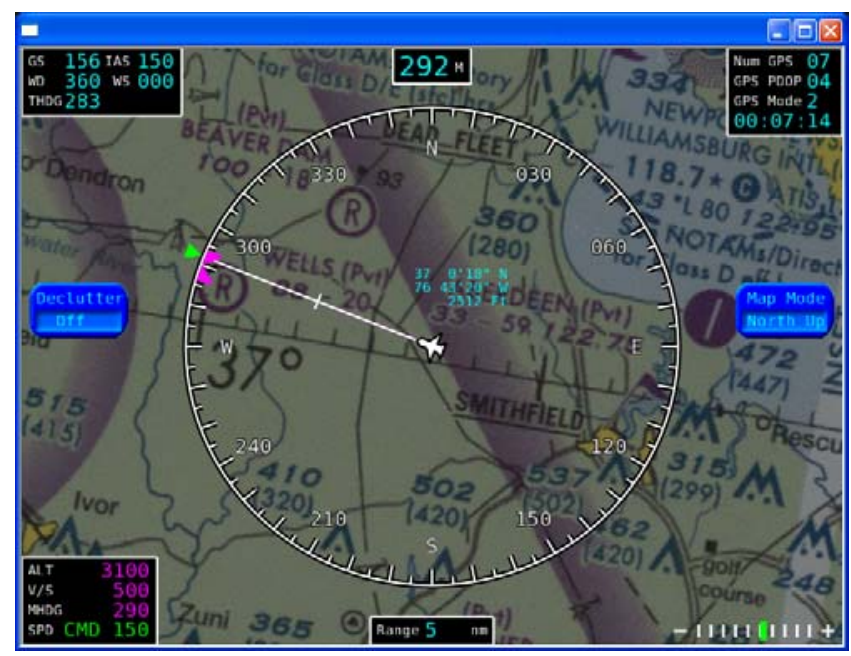

Figure 8. Moving Map Display

\section{Primary Flight Display}

Identical PFD displays are available in the Ground Station and aircraft. However, the PFD in the aircraft is only seen by the Systems Operator in the rear seat. The PFD in the rear seat provides redundant data to that seen by the Safety Pilot, since all of the standard instruments are available on the main instrument panel. The PFD has a pitch ladder and roll indicator in the center, airspeed indicator on the left, altitude ladder and vertical speed indicator on the right side. A lower left box contains ADAHRS outside air temperature, true airspeed and ground speed. Hollow 
magenta chevrons indicate preselected airspeed, altitude, and vertical speed on their respective displays. The chevrons become solid when an airspeed, altitude, or vertical speed is being commanded. The PFD is shown in Figure 9.

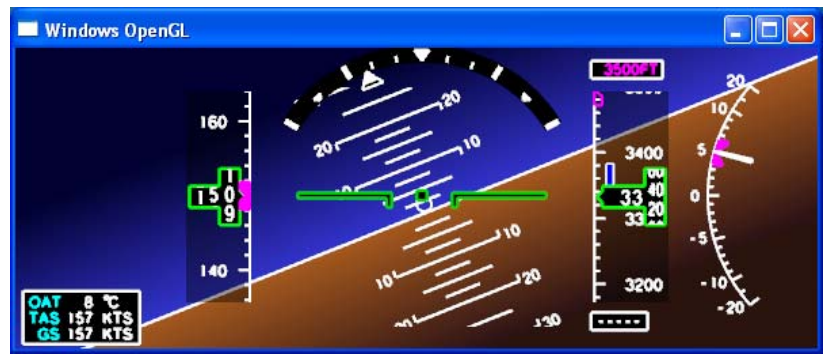

Figure 9. Primary Flight Display

\section{Simulation}

A unique simulation-to-flight capability was developed to support the SR22 surrogate UAS and future research for NASA's GA aircraft. The first phase in the development of this capability was to prepare a simulation that was representative of the SR22 test aircraft. The LaSRS++ GA simulation framework includes an adaptive aerodynamic package that was configured to approximate an SR22 aircraft. Autopilot and auto-throttle capabilities were then added. These systems were designed to be as generic as possible while still being capable of replicating the behavior of the STEC-55 autopilot and research autothrottles installed on the SR22 aircraft. The next phase involved development of hardware interfaces enabling these and other models to communicate using the same ARINC 429, discrete input/output (I/O), analogs, and RS-232 data as the aircraft systems. This capability enabled verification and validation of the surrogate UAS flight and Ground Station software using a systems integration laboratory (SIL) approach. However, limited hardware resources required sharing between the SIL and the SR22 aircraft. Furthermore, there was no dedicated GA SIL where testing could be conducted.

These restrictions led to the implementation of a simulated communications medium concept. This involved modification of the low-level hardware interfaces for ARINC 429, discrete, analog, and RS232 communications abstractions to utilize the Transmission Control Protocol/Internet Protocol (TCP/IP) communication protocol when the physical communications medium was not present. Incorporation of these modified communications abstractions into both simulation and flight/Ground Station software enabled development of a new simulation-to-flight concept which utilizes a pseudoSIL when a traditional SIL is not available, or when only portions of the actual communications media and/or hardware are available. Since the initial release of the surrogate UAS flight and Ground Station software, virtually all subsequent software releases have been successfully verified and validated in a completely pseudo-SIL environment.

\section{Data Communications}

The VHF and UHF radios are connected to the General Purpose Computer via the RS-232 serial interface operating at 9600 baud. The software does not distinguish between the VHF and UHF radios. This feature allows the VHF and UHF radios to be interchanged without software changes. Currently, only two of the three radio communications channels are used at one time. The software can be configured to use all three or more channels if needed.

\section{Aircraft Downlink Message}

The aircraft-to-Ground Station downlink message is designed to provide enough information regarding the aircraft state and status to give the Ground Station operator good situational awareness. Most of the information in the message comes from the ADAHRS and includes position, attitude, velocity, acceleration, and air data. The message length is 124 bytes for the aircraft status downlink message. The aircraft message update rate is programmable but defaults to $3 \mathrm{~Hz}$. This rate is sufficient to update the Ground Station displays to give the Ground Station operator good awareness of what the UAS Surrogate is doing without exceeding the radio bandwidth or duty cycle. The computer sends messages to the radios at $9600 \mathrm{bits} / \mathrm{sec}$ or $0.1 \mathrm{msec} / \mathrm{bit}$. A 124-byte message contains approximately 1240 bits at ten bits per character. Transmission time for one 124-byte message is approximately $130 \mathrm{msec}$. Therefore, a maximum of seven air-to-ground message updates could be sent per 1-sec period. The $3 \mathrm{~Hz}$ update rate corresponds to a $39 \%$ duty cycle. At this duty cycle, $61 \%$ of the time is available to receive ground-to-air commands from the Ground Station. The aircraft 
status information contained in each downlink message is listed in Table 1.

Table 1. Aircraft Downlink Status Message

\begin{tabular}{|l|l|l|}
\hline Parameter & Range & Bits \\
\hline Start Sequence & “AbCd" & 32 bits \\
\hline Remaining Bytes & 120 & 32 bits \\
\hline Checksum & 0 to 2 32 -1 & 32 bits \\
\hline Validity Bits & FFFFFFFF & 32 bits \\
\hline Latitude deg & -90 to +90 & 32 bits \\
\hline Longitude deg & -180 to +180 & 32 bits \\
\hline Altitude ft & $-1 \mathrm{k}$ to $+60 \mathrm{k}$ & 32 bits \\
\hline Roll deg & -90 to +90 & 32 bits \\
\hline Pitch deg & -90 to +90 & 32 bits \\
\hline Heading M deg & 0 to 360 & 32 bits \\
\hline Heading T deg & 0 to 360 & 32 bits \\
\hline Indicated Air Speed & 0 to 800.8 & 32 bits \\
\hline True Air Speed & 0 to 800.8 & 32 bits \\
\hline Ground Speed & 0 to 800.8 & 32 bits \\
\hline Vertical Speed ft/m & +-10000 & 32 bits \\
\hline M Track deg & 0 to 360 & 32 bits \\
\hline Flight Path Angle d & -90 to +90 & 32 bits \\
\hline Turn Rate deg/s & -200 to +200 & 32 bits \\
\hline Lat Acceleration g & -10 to +10 & 32 bits \\
\hline Wind Speed kts & 0 to +97.192 & 32 bits \\
\hline Wind Direction deg & -180 to +180 & 32 bits \\
\hline Outside Air T deg C & -70 to +70 & 32 bits \\
\hline GPS Time of Week & 0 to 86400 & 32 bits \\
\hline GPS Sats Tracked & 0 to 32 & 32 bits \\
\hline GPS PDOP & 0 to 100 & 32 bits \\
\hline GPS MODE & 0 to 4 & 32 bits \\
\hline Heading Command & 0 to 360 & 32 bits \\
\hline Altitude Command & 0 to 12000 & 32 bits \\
\hline Vertical Speed Com & -700 to +700 & 32 bits \\
\hline Airspeed Command & 80 to 250 & 32 bits \\
\hline Engaged Modes & Discrete Bits & 16 bits \\
\hline Padding & $0 x 00 ~ 00$ & 16 bits \\
\hline & & \\
\hline
\end{tabular}

\section{Ground Station Uplink Message}

The Ground Station uplink messages contain the four commands, synchronization information, a sequence number, mode/status information and a checksum. The uplink messages are sent only when there is a new command issued by the Ground Station operator. These messages are much shorter in length at 32 bytes and one message can be transmitted in approximately $33.3 \mathrm{msec}$. Uplink command messages are sent once each from the two Ground Station radios before listening for a return from the airborne system. The four commands that are uplinked to the aircraft are returned to the Ground Station by the aircraft system to serve as confirmation for the uplinked commands and for display on the Ground Station moving map display. The information contained in each uplink command message is listed in Table 2.

Table 2. Ground Station Uplink Message

\begin{tabular}{|l|l|l|}
\hline Parameter & Range & Bits \\
\hline Start Sequence & “AbCd” & 32 bit \\
\hline Remaining Bytes & 28 & 32 bit \\
\hline Checksum & 0 to $2^{\wedge} 32$-1 & 32 bit \\
\hline Heading Command & 0 to 360 & 32 bit \\
\hline Altitude Command & 0 to 12000 & 32 bit \\
\hline Vertical Speed Com & -700 to +700 & 32 bit \\
\hline Airspeed Command & 80 to 200 & 32 bits \\
\hline Sequence Number & 0 to 65535 & 16 bits \\
\hline Selected Modes & Packed Bits & 8 bits \\
\hline Padding & $0 x 00$ & 8 bits \\
\hline
\end{tabular}

\section{Error Detection}

A 32-bit checksum is computed and inserted into each downlink and uplink message. The checksum is received and verified by the respective aircraft or Ground Station software. Any message that fails the checksum test is rejected. A sequence number is also inserted in the uplink message. The aircraft software uses the sequence number to distinguish between new, old or redundant messages. Old or redundant messages are discarded by the aircraft software.

\section{The UAS Ground Station}

\section{Components}

The Ground Station uses a nearly identical computer to the one installed in the aircraft. However, the only required interface is the RS-232 serial and Ethernet links to the VHF and UHF data radios. The Ground Station computer has dual video outputs for dual displays, although only one is required. The dual display configuration allows more 
separation and arrangement of the various display elements. These display elements include the moving map display, a PFD, and a GUI panel for entering the heading, altitude, vertical speed, and airspeed commands. Additional GUI panels can be activated by the Ground Station operator to display state and status information residing in the computer or sent from the aircraft. The Ground Station computer configuration is shown in Figure 10.

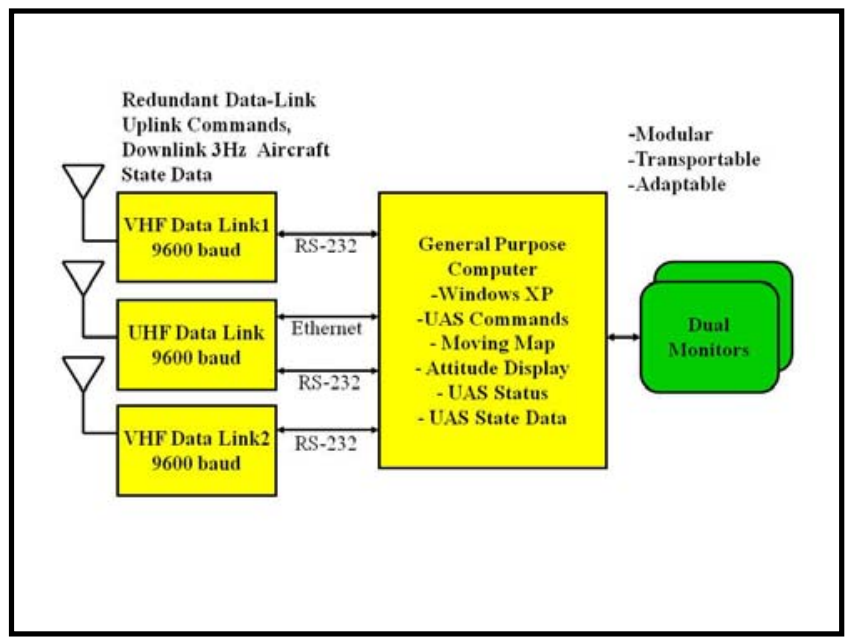

Figure 10. Ground Station Components

\section{Portability}

The Ground Station was designed to be portable. The UAS Ground Station consists of a few lightweight components including a 20-lbs PC, two 9-lbs display monitors, two or three radios weighing 1.2 lbs each, and the antennas. The antennas are potentially the least transportable part of the system. Several different VHF and UHF antennas have been used for the project with some of them small and light weight and others large and heavy. Currently, all of the Ground Station antennas are installed on the roof of a $100-\mathrm{ft}$ high aircraft hangar. Originally, several hundred feet of coaxial cable were required to connect the radios to the roof antennas with corresponding large signal losses. For this reason, the radios subsequently were located near the roof antennas with short coaxial cable runs to minimize losses. Fiber optic RS-232 converters were used to connect the radios to the Ground Station computer. Power is available in the hangar roof to power the radios and fiber optic converters. Power and the ability to install hundreds of feet of fiber or coaxial cable may not exist at a future ground station site.
Therefore, the portability of the ground station is site specific and requires detailed knowledge of the site. Site data include building configuration, power availability, capability to run cables and mount antennas, geographic terrain, flight profiles and many other considerations.

\section{Flight Tests}

\section{Flight Test Procedures}

A flight test procedure was developed and approved by the NASA Langley Airworthiness and Safety Review Board (ASRB). Safety procedures included preflight briefings for all participants, a Safety Pilot specific checklist, prepared flight test cards, flight operations limited to day visual meteorological conditions (VMC), and no modified autopilot engagement below $500 \mathrm{ft}$ of altitude. Other safety features include Safety Pilot's ability to manually override the autopilot and autothrottle, autopilot disconnect switch mounted on the side arm controller, easily accessible autopilot circuit breaker, and the ability to disable all research power with one switch on the center console. A separate alternator that provides up to 50 A of 28 Volt DC power to the research computer and all research equipment provides the Cirrus research power. The UAS cockpit controls and displays are shown in Figure 11.

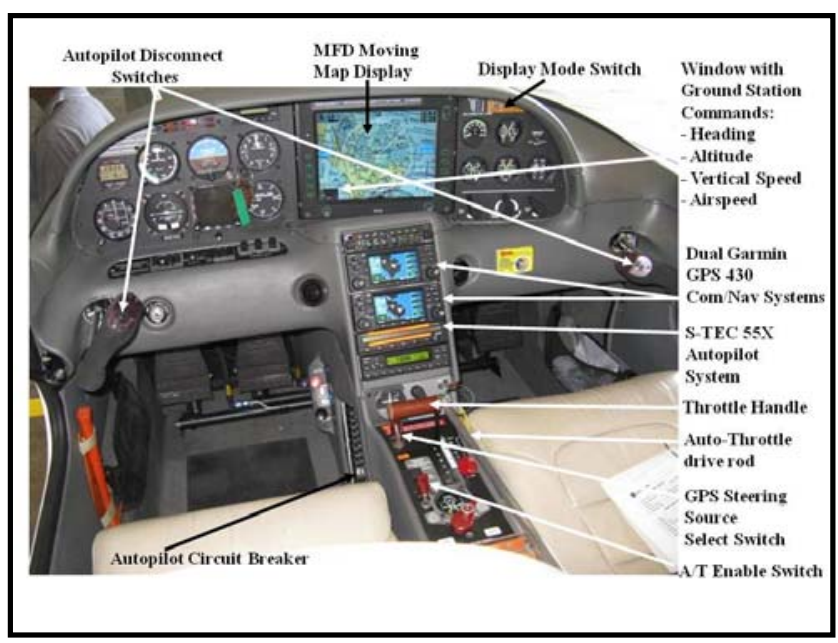

Figure 11. UAS Cockpit Controls and Displays

To date, all UAS Surrogate flight tests have been local to the NASA Langley/Langley Air Force Base area. All UAS flights were preceded by ground 
preflight checks of the aircraft and Ground Station systems including the UAS command/status radio links. After takeoff and above $500 \mathrm{ft}$ altitude, the procedure allowed engaging the autopilot, autothrottle and enabling commands from the Ground Station. All Ground Station commands were coordinated between the Safety Pilot and Ground Station operator via VHF voice communications. Each command sent by the Ground Station was acknowledged by the Safety Pilot and echoed back to the Ground Station by the aircraft downlink message.

\section{Performance}

The goal of remotely controlling the aircraft in all three axes has been achieved with line of sight range limitations. The Ground Station operator may routinely fly the aircraft with heading, altitude, vertical speed and airspeed commands. The flight performance of the UAS Surrogate has been variable depending on conditions. The aircraft response varies with such factors as airspeed and turbulence. Above about 148 knots of airspeed, the autopilot roll authority is reduced due to the greater aerodynamic forces on the control surfaces. The roll servo upgrade should solve this and other limitations, but was not tested before this paper was submitted.

\section{Future Plans}

\section{Satellite Communications}

The current VHF and UHF data-link radios are limited to operations within 30-40 n. mi. of the Ground Station. It is desirable to operate the UAS Surrogate beyond visual range with the addition of a satellite communications system. This satellite system must be capable of real-time voice and data communications. Several companies offering some form of satellite communications services include Iridium, Globalstar, and Inmarsat. The currently available first generation satellite systems all suffer from many limitations, including low data rate, long propagation delays, low connection reliability, and high cost. Several old and new satellite companies have upgrade plans to address many of these issues. TerreStar, Skyterra, ICO Global Communications, Globalstar, and Iridium and others are developing these new or upgraded geostationary (GEO), low earth orbit (LEO) and hybrid systems. These next generation satellite communications systems should offer a host of new products and services with upgraded broadband data rates from $250 \mathrm{~Kb} / \mathrm{sec}$ to 1 $\mathrm{Mb} / \mathrm{sec}$. These new services are predicted to expand into the aeronautics market. The number of aeronautical satellite communications terminals alone is predicted to grow from 37,000 units in 2009 to over 72,000 by 2018 [7]. There is also a developing convergence of communications technologies. The new TerreStar Genus phone, developed by TerreStar, AT\&T, and Qualcomm is the first smart phone to communicate with cellular and satellite networks [8]. The phone also has Wi-Fi and 3G Internet capabilities with service to begin in 2010. These and other emerging technologies may make it possible for UAS Surrogate operations anywhere in the North American coverage area.

\section{Waypoint Navigation}

Another future upgrade phase planned for the UAS Surrogate is a waypoint navigation feature. This upgrade will give the Ground Station operator the capability to build, modify, and upload to the aircraft a flight plan containing a series of navigation waypoints. The UAS Surrogate will be able to automatically fly the flight plan including heading, altitude and speed changes.

\section{Conclusions}

The UAS Surrogate project has demonstrated that a GA aircraft can be made into a remotely controlled aircraft. It has also shown that a UAS Surrogate could be a platform to perform research and testing of UAS systems. The file-and-fly capability has been demonstrated and will allow the UAS Surrogate to perform a variety UAS research in the NAS. However, several problem areas were revealed that must be addressed before routine UAS operations in the NAS can be realized. A major issue is radio communications. Specifically, how to provide secure, reliable, dedicated, and redundant communications? There is a potential for disaster when UAS command-and-control communications are lost. Another problem area is poor radio performance on the growing number of UAS vehicles and aircraft manufactured from composites. During this UAS Surrogate development, considerable time and effort went into solving problems with radio data-link 
communications including radio power, interference, EMI, and antenna performance. These areas are just a few of those that need to be addressed, along with the widely-known UAS/NAS integration problems of traffic sense and avoid, air traffic control (ATC), ATC-UAS communications, and standards development. Although many people and institutions are working on these and other UAS related problems, there is room for more research.

\section{References}

[1] Flynn, Frances P., William M. Moran, 1984, Spectrum Resource Assessment In The $162-174 \mathrm{MHz}$ Frequency Band, NTIA Report 84-143, Washington DC, U. S. Department Of Commerce, pp. 3.

[2] Fink, Donald G., Alexander A. McKenzie, 1975, Electronic Engineers Handbook, First Edition, New York, McGraw-Hill Inc., Chapter 18, pp. 20-22.

[3] Tooley, Mike, David Wyatt, 2009, Aircraft Electrical and Electronic Systems, Principles, Maintenance and Operation, First Edition, Great Briton, Elsevier Ltd., pp. 344.

[4] Scott, Dean A., 2007, A High Performance Airband Antenna For Your Ultralight/Lightsport Aircraft, March 19, 2007, Chrusion.com, http://chrusion.com/BJ7/InvVeeAntenna4ULs.pdf

[5] Fink, Donald G., Alexander A. McKenzie, 1975, Electronic Engineers Handbook, First Edition, New York, McGraw-Hill Inc., Chapter 18, pp. 62.

[6] Buchsbaum, Walter H., 1978, Buchsbaum's Complete Handbook of Practical Electronic Reference Data, Second Edition, Englewood Cliffs, NJ, Prentice Hall Inc., pp. 8.

[7] Li, W., 2010, Growth On The Horizon For MSS Sector, May, Sonoma, CA, SatMagazine, pp. 16-20.

[8] Epstein, Jeff, 2009, TerreStar, December, Sonoma, CA, SatMagazine, pp. 156-157.

29th Digital Avionics Systems Conference

October 3-7, 2010 